\title{
Assessment of Stroke Patients' Status and Their Transfer Time by Emergency Medical Services to Valiasr Hospital in Arak, Iran
}

\author{
Saiedeh Bahrampouri' ${ }^{1}$, Hamid Reza Khankeh ${ }^{2,3^{*}}$, Asghar Dalvandi
}

1. Department of Health in Emergency and Disaster Research, University of Social Welfare \& Rehabilitation Sciences, Tehran, Iran

2. Department of Nursing, University of Social Welfare \& Rehabilitation Sciences, Tehran, Iran.

3. Department of Clinical Science and Education, Karolinska Institutet, Solnavägen, Solna, Sweden.

Citation: Bahrampouri S, Khankeh HR, Dalvandi A. Assessment of stroke patients' status and their transfer time by emergency medical services to Valiasr Hospital in Arak, Iran. Health in Emergencies and Disasters Quarterly. 2016; 1(3):163-172. http://dx.crossref.org/10.15412/J.HDQ.09010307

\section{: http://dx.crossref.org/10.15412/J.HDQ.09010307}

Article info:

Received: 05 Nov. 2015

Accepted: 11 Feb. 2016

\section{Keywords:}

Diagnosis of status, Transfer time, Stroke patient, Prehospital emergency

\section{A B S T RACT}

Background: Stroke is a major cause of death and disability in the world and these complications can be decreased by competent emergency care. Emergency medical service (EMS) transfer half of the stroke patients to hospitals. This study aimed to determine diagnosis of status and transfer time of stroke patients by EMS to a main hospital (Valiasr) in Arak City.

Materials and Methods: This study was a descriptive analytic study and conducted on 43 patients with a diagnosis of stroke that transferred by EMS to Valiasr Hospital in Arak City. Data were collected through a checklist which contained information about age, sex, type of accident, response time, scene time, transfer time, and total time from inpatients records and Emergency Center statistics. Regarding data analysis, descriptive statistical tests were performed by SPSS 19.

Results: The mean(SD) age of patients was $73.7(3.8)$ years and $51.2 \%$ of them were women. The stroke diagnosis by EMS technicians was correct in $15(34.9 \%)$ cases. In $20(46.5 \%)$ cases, the diagnosis was wrong and in $8(18.6 \%)$ cases, there were not any diagnosis. The most common non-stroke condition was confusion. The mean response time and arrival time to location, transfer time, and total time were $6.9,16.9,9.1$, and 35.3 minutes, respectively. In patients with correct diagnosis and wrong or no diagnosis, these mean times were 7, 17.1, 3.9, and 35.7 minutes and 6.9, 16.8, 9.7, and 33.5 minutes, respectively.

Conclusion: The results of this study showed that an accurate diagnosis by EMS technicians could be an indicator for faster transfer of the patient to treatment center. It is recommended that more appropriate pre-hospital diagnosis tools of stroke be developed and handed to EMS staff, for more accurate diagnosis of strokes and their better treatment. 


\section{Introduction}

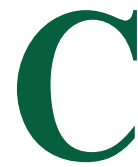

erebrovascular disease is the third common cause of death, and the sixth leading cause of disability in the world and expected to rise to the fourth cause of disability and second leading cause of death by 2020

[1]. Today, stroke has many treatments but role of time is critical in returning blood to brain and acute stroke care has significantly reduced death and disability rate among these patients [2, 3]. Emergency medical services (EMS) is the first medical contact for half of the acute stroke patients. Thus, they play an undeniable role in survival and prevention of complications in these patients [4]. Getting "the right patient to the right place in time" is the key to save lives and reduce disability caused by stroke [5].

The annual incidence of stroke is 15 million worldwide. Over 5.5 million of these patients will eventually die and over two-thirds of all stroke deaths in the world occur in developing countries [6, 7]. The annual incidence of stroke per 100000 people in Western countries is 100 to 300 while in Iran, based on some altered studies, average annual stroke attack rates are between 33 to 372 per 100000 [8]. The case fatality rate at 28 days for all type of strokes is reported as $12.6 \%$ in the world and about $19 \%$ to $31.5 \%$ in Iran $[9,10]$.

Age is a known key factor in the incidence of stroke. The risk of stroke doubles for each successive decade after age 55 [3]. The annual incidence of elderly stroke (over 45 years) was 500 per 100000 people [11]. Also, stroke reduces mobility in more than half of stroke survivors whose age is 65 and older [12]. Today, stroke has been considered one of the most costly diseases because of acute and chronic disabilities resulting from stroke [9].

A stroke is an interruption of cerebral circulation, occurs when a clot blocks the blood supply to the brain or a blood vessel in the brain bursts $[13,14]$. Stroke symptoms may begin with central nervous system function failure [15]. They include sudden weakness or numbness of the face, arm, or leg, especially on one side of the body; sudden confusion; trouble in speaking or understanding; sudden trouble in seeing in one or both eyes; sudden trouble in walking, dizziness, loss of balance or coordination; or sudden severe headache with no known cause [16]. Stroke signs and symptoms encompass a wide range of neurological symptoms, which may also be seen in other central nervous system disorders. These diseases include epilepsy, diabetes dementia, brain tumor, and abscess. The most common stroke symptoms mimic is seizure $[17,18]$.
Today, there are different treatments of stroke. All efforts should be laid on immediate symptom recognition, rapid transport, and treatment [19]. Several studies have shown that the effectiveness of any stroke therapy is time dependent [19]. Because with passing every minute of the onset of untreated stroke, have died 200 million nerve cells [20]. The treatment is dependent on time, i.e. the difference between returning to work or becoming permanently disabled at home or in a nursing home [5].

The last decade of stroke studies has highlighted the importance of rapid treatment of acute stroke [21, 22]. Research has shown that speed, knowledge, and technology would affect prevention, recognition, and transportation to treatment, post-treatment, and rehabilitation. They can make a vast difference in stroke victim's outcome [20]. In this regard, mortality rates in the first 30 days after stroke have decreased because of advances in emergency medicine care [10].

American Heart Association explained the best care for stroke patients with 7 Ds. These "7 Ds of Stroke Care" include detection (recognition of stroke signs and symptoms by the patient and family), dispatch (call 115 and priority EMS dispatch), delivery (prompt transport and pre-hospital notification to hospital), door (arrival and urgent triage in the emergency department), data (ED evaluation, prompt laboratory studies, and CT imaging), decision (diagnosis and decision about appropriate therapy), and drug (administration of appropriate drugs or other interventions) $[23,24]$. Thus, both pre-hospital and hospital services take part in the treatment chain of stroke patients [19]. Pre-hospital phase is considered as an interval between manifestation of symptoms and reaching the appropriate hospital and has two parts; stroke symptom recognition by the patient and family and transferring to medical center by EMS [25].

Pre-hospital emergency medical services respond to call for help, assess the medical needs of the patient, provide health service in difficult environments outside the hospital and transport the sick or injured people to appropriate medical facilities [15]. In other words, EMS links hospitals and other medical centers to community, attempts to provide required emergency information and services in a suitable method and in a short time, and take the patients to the nearest medical center to prevent serious complications that can threat their lives [26]. Paramedics are the first medical contact in $38 \%$ to $70 \%$ of stroke patients and maximum delay in the assessment and treatment of stroke occurs in pre-hospital phase [13, 27, 28]. Thus, emergency medical technicians play an important role in the management of patients in acute phase as well 
as an essential part in stroke treatment team [29, 30]. Adams et al. reported that the benefits of EMS activities for patients with stroke symptoms appear in both the pre-hospital and in-hospital settings. EMS is strongly associated with decreased transfer time and prevention of neurological deficits in hospitals [23].

The objectives of the EMS phase of stroke care are as follows: 1) rapid and accurate diagnosis of stroke as the cause of the patient's problems, 2) elimination of comorbid conditions that could mimic stroke (e.g. epilepsy, diabetes dementia, brain tumor, and abscess) [18, 27], 3) stabilization of patients' condition, 4) rapid transfer of the patient to the closest appropriate emergency department, and 5) notification of the receiving institution about impending arrival of a patient with suspected stroke [23].

Transferring by EMS is the most important factor in reducing delays in reaching the hospital [31]. In dealing with patients outside of the hospital, use of a standardized protocol helps ensure that the major components of a neurological examination are performed in a timely fashion [17]. They can reduce the response time of the pre-hospital care of these patients [17]. Common standard protocols for stroke diagnosis in the world are FAST (Face, Arm, Speech, Test), CPSS (Cincinnati Prehospital Stroke Scale), and LAPSS (Los Angeles Prehospital Stroke Screen) [13, $23,32]$. Chenkin et al. following the implementation of a diagnostic protocol in a city, witnessed the increased number of stroke patients' eligible to receive tissue Plasminogen Activator (tPA) to $17.2 \%$ [33].

Also, Harbison and Ramanujam studies showed that $40 \%$ to $79 \%$ of stroke patients are diagnosed correctly by pre-hospital emergency technicians [34, 35]. The results of studies in America, Australia, and Italy showed that the percentage of positive results with the drug tPA was exceeded if the patients be transferred by EMS [36]. Therefore, the correct diagnosis of stroke by emergency medical technicians can reduce transferring delays from the onset symptoms to hospital admission, also can prevent losing treatment resources [16, 34, 37, 38].

Success in EMS depends on various factors, including responsible and trained personnel and coordinated communication system [32]. Thus, current situation should be assessed to enhance and strengthen the quality of services performed by the pre-hospital emergency care [32, 39]. Three important criteria in this evaluation include the level of personnel training, equipment, and response time interval [39]. Response time can be divided into response time, time at the scene, and transported time to hospital [39].
This study has focused on the pre-hospital stroke diagnosis because of its importance and paucity of research in the field. Also, we tried to identify existing deficiencies, and if possible, resolve them.

In the end, it seems that accurate pre-hospital diagnosis and transfer time and relationship between these 2 variables, could depict a clear picture of the current management of stroke patients in pre-hospital phase.

\section{Materials and Methods}

This cross-sectional study used a descriptive analytical design and conducted on stroke patients transported by EMS to Valiasr Hospital in Arak City from 23 August 2010 to 23 August 2011. Patients with a final diagnosis of stroke in hospital were divided into 3 groups of correct diagnosis, misdiagnosis, and no diagnosis of stroke. Studied variables included response time, time at the scene, transfer time, and total time. The study population included consecutive patients transported to Valiasr Hospital by EMS in Arak City over a one-year period.

In order to increase the validity of the research, study was a census of all individuals who met the inclusion criteria. Inclusion criteria were a final hospital-based diagnosis of stroke, matching patient name in hospital record sheets and EMS record sheets, and being transported by Arak City EMS to Valiasr Hospital. Patients with missing data or incomplete full name were excluded from the main analysis.

Researchers after receiving institutional ethical approval, referred to Arak City EMS and Valiasr Hospital, explained the research objectives and emphasized on data confidentiality. Finally, they came to an agreement and started to collect the data. In addition to ethical considerations, names of all patients who met the inclusion criteria were removed and numeric codes assigned to them.

In brief, Arak City EMS delivered services to 599634 people in Arak. Also Arak City EMS has 7 urban-based and 6 road-based centers and covers an area of $7178 \mathrm{~km}^{2}$ [40, 41]. Valiasr Hospital (the only stroke state center in Markazi Province) admits all stroke patients that transfer by Arak City EMS.

At first, names of all patients with a final diagnosis of stroke were extracted from the patients' records of Valiasr Hospital from 23 August 2010 to 23 August 2011.

The final diagnosis was defined as a final inpatient discharge diagnosis of stroke or CVA that was recorded in patients' file by neurologist. The final diagnosis by neu- 
rologist is based on computed tomography (as standard clinical criteria for stroke diagnosis). A total of 231 patients were selected in the sample. In the end, hospital records of all stroke patients were assessed and the cases transferred by EMS were identified.

Based on the records, 57 people by Arak EMS, 47 people from other cities, and 3 people from other hospitals in Arak City were transferred to Valiasr Hospital. Our research only examined the patients that transferred by Arak EMS.

Then, researchers referred to statistical center of Arak EMS, and received stroke patients' data, including times intervals, first diagnosis, and demographic characteristics of the patients. In this phase, 14 samples were omitted because of not having records by technicians and not matching in 2 centers. Finally, the study population comprised 43 people with final diagnosis of stroke who were transferred by Arak City EMS.

Tools for data gathering in this study was a researchermade checklist comprising age, sex, primary diagnose, time received, time of reaching to scene, time of departure from the scene, and time of arrival at the hospital. This information was completed by the researchers. Based on above information, response time, time at the scene, transport time and total run time were calculated (Table 1) [42].

All statistical analyses were conducted with SPSS version 19. The demographic characteristics and diagnosis status for the study population were compared by $\chi^{2}$ test. Then, we used Mann-Whitney test for assessing the relationship between diagnosis status and time intervals. Mean time intervals ( $95 \%$ confidence interval) were calculated, too. A value of $\mathrm{P}<0.05$ was considered statistically significant for all tests.

\section{Results}

During the study period, out of 231 patients identified with stroke diagnosis, 43 patients were included in this study, because they were transported by Arak City EMS and hospitalized in Valiasr Hospital based on final diagnosis of stroke by physicians and inclusion criteria.

Most patients were older than 55 years (93\%) and were females $(51.2 \%)$. Stroke frequency in age $75-85$ years in both sexes, males less than 55 years and women aged between 55 to 65 years and 75 to 85 years old were further and did not see significant difference in other ages. The mean(SD) age of the study population was 73.7(3.8) years, with the range of 38 to 100 years and the mean age of men and women were 72 and 75 years, respectively.

Out of 43 patients, $15(34.9 \%)$ had a correct diagnosis, $20(46.5 \%)$ had a wrong diagnosis, and $8(18.6 \%)$ did not have a diagnosis by EMS technicians. The mean age of the subjects were 69.4 years at correct diagnosis group, 75.9 years at misdiagnosis group, and 76.6 years at no diagnosis group.

Figure 1 indicates the types of diagnosis for stroke patients by EMS technicians. Confusion was the most prevalent misdiagnosed disease (28\%), followed by seizures (5\%), weakness $(5.1 \%)$, and other misdiagnoses (including respiratory problems, infections, dizziness, and other neurological problems) (9\%). Time intervals (response time, time at the scene, transfer time and total time) for all study population are given in Table 2.

Overall, the average age of patients who were misdiagnosed or had no diagnosis was 77 years and the average age of patients with a correct diagnosis was 69 years.

Table 4 presents the demographic characteristics of the diagnostic status of sample. The results indicate that no statistically significant differences were found among correct, wrong, or without diagnosis of stroke patients by EMS technicians with regard to age and sex.

Table 5 indicates that response time, time at the scene, and total time for patients with stroke did not vary significantly in both groups (correct and wrong or without diagnosis). Also on $68.4 \%$ of stroke patients, the response

Table 1. Time interval definition.

\begin{tabular}{cc}
\hline Time intervals & Definition \\
\hline Response time & Period between the emergency call received and ambulance arrival at the scene \\
\hline Time at the scene & Interval between leaving the scene and arriving at the emergency department \\
Transport time & Response time, scene time, and transport time which amount to the total run time \\
Total run time & IHlealth in \\
\hline
\end{tabular}




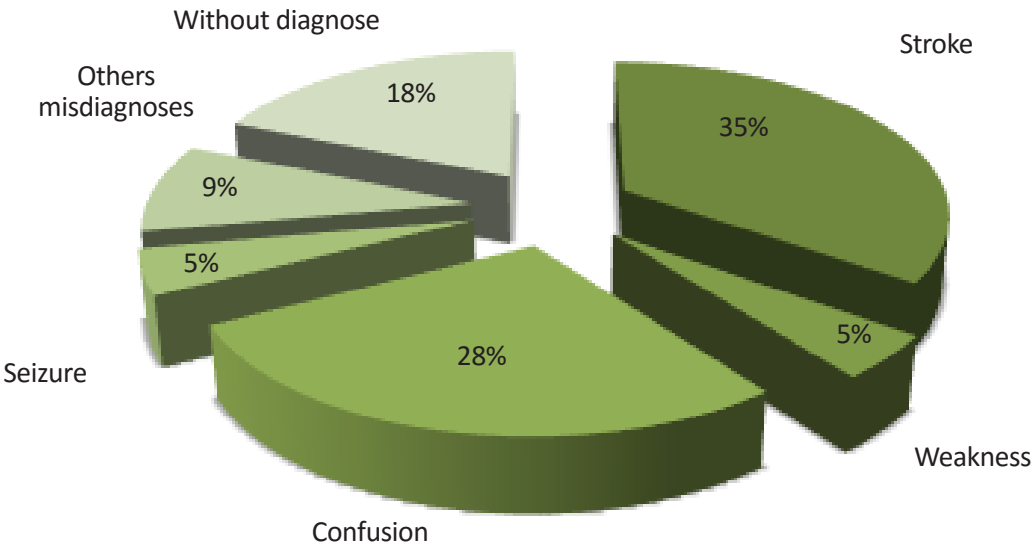

Figure 1. Frequency of stroke diagnosis by EMS technicians.

Table 2. Time intervals (min) for all study population.

\begin{tabular}{ccc}
\hline Time intervals & Mean time & Range \\
\hline Response time & 6.9 & $15.6-2.4$ \\
& $1.06 \pm 7$ & 16.9 \\
Time at the scene & $2 \pm 17$ & $31.5-8.9$ \\
Transport time & 9.1 & $58.1-0.6$ \\
Total run time & $3.6 \pm 9.3$ & $92.3-18.5$ \\
\hline & $4.6 \pm 35.1$ & 9 \\
\hline
\end{tabular}

Table 3. Times intervals (min) with respect to type of diagnosis by EMS technicians.

\begin{tabular}{|c|c|c|c|c|}
\hline Type of diagnosis & Time interval & Mean time & Range & SD \\
\hline \multirow{4}{*}{ Correct diagnosis } & Response time & 7 & $15.6-2.4$ & 3.8 \\
\hline & Time at the scene & 17.1 & $26.7-9.8$ & 5.7 \\
\hline & Transport time & 3.9 & $9.3-0.9$ & 2.5 \\
\hline & Total run time & 35.7 & $87.3-20.2$ & 17.3 \\
\hline \multirow{4}{*}{ Wrong diagnosis } & Response time & 6.5 & $13.6-2.6$ & 2.8 \\
\hline & Time at the scene & 17 & $31.5-9.3$ & 5.8 \\
\hline & Transport time & 12.1 & $58.1-2.4$ & 15.6 \\
\hline & Total run time & 35.7 & $92.3-18.5$ & 18.2 \\
\hline \multirow{4}{*}{ Without diagnosis } & Response time & 7.7 & $10.4-4.2$ & 1.9 \\
\hline & Time at the scene & 16.5 & $26.8-8.9$ & 6.1 \\
\hline & Transport time & 9.1 & $21.9-0.6$ & 6.8 \\
\hline & Total run time & 33.3 & $55.7-22.1$ & 10 \\
\hline
\end{tabular}


Table 4. Demographic characteristics of 2 groups (with correct and wrong or without diagnosis) by EMS technicians.

\begin{tabular}{|c|c|c|c|c|}
\hline & \multirow[b]{2}{*}{ Variable } & \multicolumn{2}{|c|}{ Diagnosis status } & \multirow[b]{2}{*}{ P-value } \\
\hline & & $\begin{array}{l}\text { Correct diagnosis } \\
\text { Frequency (\%) }\end{array}$ & $\begin{array}{l}\text { Wrong or without diagnosis } \\
\text { Frequency (\%) }\end{array}$ & \\
\hline \multirow{2}{*}{ Age } & $<60$ years & $4(27)$ & $3(10.7)$ & \multirow{2}{*}{0.18} \\
\hline & $\geq 60$ years & $11(73)$ & $25(89.3)$ & \\
\hline \multirow{2}{*}{ Sex } & Female & $6(40)$ & $16(57)$ & \multirow{2}{*}{0.29} \\
\hline & Male & $9(60)$ & $12(43)$ & \\
\hline
\end{tabular}

Table 5. Relationship between time intervals of 2 groups (with correct and wrong or without diagnosis) by EMS technicians.

\begin{tabular}{|c|c|c|c|}
\hline \multirow[b]{2}{*}{ Variable } & \multicolumn{2}{|c|}{ Diagnosis } & \multirow[b]{2}{*}{ P-value } \\
\hline & $\begin{array}{l}\text { Correct diagnosis } \\
\text { Mean } \pm S D\end{array}$ & $\begin{array}{l}\text { Wrong or without diagnosis } \\
\text { Mean } \pm \text { SD }\end{array}$ & \\
\hline Response time & $7 \pm 3.8$ & $6.9 \pm 2.5$ & 0.6 \\
\hline Time at the scene & $17.1 \pm 5.7$ & $16.8 \pm 5.8$ & 0.8 \\
\hline Transport time & $3.9 \pm 2.4$ & $13.3 \pm 11.2$ & 0.01 \\
\hline Total run time & $35.7 \pm 17.3$ & $35 \pm 16$ & 0.9 \\
\hline
\end{tabular}

time was less than 8 minutes long, in which stroke patients had a mean age 73.6 years and $57.7 \%$ were female.

\section{Discussion}

Study of 231 stroke patients in the hospital during this period indicated that $25 \%$ of stroke patients were transported to hospital by EMS. Kleindorfer and Lacy in their studies showed that $38 \%$ and two thirds of stroke patients were transported to hospitals by EMS, respectively [43, 44]. Also, transporting stroke patients allocated $1 \%$ of emergency accidents in Arak. Findings of Mosley and Zhang et al. showed that $44 \%$ and $39.5 \%$ of patients were serviced by EMS. Our result was greatly lower than other similar studies [45, 46]. Low utilization of EMS in Arak is the result of weak public awareness about critical time for stroke treatment and lack of trust to EMS. Therefore, we suggest that people be educated of the signs and symptoms of stroke, transfer of the patient to the appropriate medical facility be expedite, and suitable strategy be chosen to increase public confidence on EMS with the help of mass media.

Stroke patients transferred by EMS are more women. The results of stroke frequency in women were similar to those of Ghandehari et al., which revealed stroke incidence in women as $51 \%$ to $53 \%$ [9]. Also other studies supporting the results of our study [43, 47-49]. Thus, as the incidence of stroke is higher in women; most of them are being taken to hospitals by EMS. Higher incidence of stroke among women can be due to their more attention to new problems.

The mean(SD) age of the study population was 73.7(3.8) years, which is higher than the value reported by Kothari et al. as 67(16) years and Frendl et al. as 72 years. Also in Iran, the stroke patients' age was reported around 61 to 65 years $[8,48,50]$. These results indicate need to pay more attention to diagnosis and treatment for the elderly people. Because of any disability in older age may reduce the patient's independence and lead to secondary problems for patients and their families.

Age group distribution for the sample was 9.3\% in less than 60 years people and $90.7 \%$ in higher than 60 years people. In Halpern study, patients aged higher than 45 years were $86 \%$ and in Fonarow study, the reported ages of stroke patients were $22.6 \%$ in under 60 and $77.4 \%$ in 60 years or older [ 49 , 51]. Another study by Chen et al. reported that $50 \%$ of stroke patients were more than 70 years old and the $25 \%$ of them were older than 80 years [52]. In summary, stroke incidence in patients older than 60 years was high, which was consistent with other related studies in the literature.

This study demonstrated that only $34.9 \%$ of stroke patients were correctly identified by EMS technicians. However, According to some authors, diagnosis accuracy reported as $72 \%$ by Kothari, $40 \%$ by Ramanujam and with utilization 
of diagnostic tools, $67.7 \%$ by Andjelic, $77 \%$ by Smith for paramedics who did not use any diagnostic tools, and 79\% by Harbison for paramedics who applied FAST [34, 35, $48,53,54]$. Therefore, poor diagnosis of stroke patients accounts for increase in pre-hospital delay, on the other hand, correct diagnosis of stroke reduces mortality and disability in patients [55]. Moreover, any attempt to improve the accuracy of diagnosis by EMS technicians can improve the emergency treatment process.

The results of this study suggest that older patients have higher chance for wrong diagnosis (69 vs. 76 years). In our study, weakness, confusion, seizures, respiratory problems, infectious causes, vertigo, and other neurological problems (46.5\%) were associated with stroke misdiagnosis by EMS technicians. According to some authors (Kothari and Harbison), the main disorders that misdiagnosed with stroke were infectious causes, including sepsis, pneumonia, urinary tract infection, and encephalitis; myocardial infarction and syncope; seizures; confusion; malignant tumors; psychological disorders; dementia; subdural hemorrhage; drug and alcohol abuse; and peripheral neuropathy [48]. This issue can demonstrate that EMS technicians are not able to distinguish between stroke and mimics.

In our study, the mean response time was 6.9 (median=6.7) minutes. However, previous studies reported that time as 13 and 6.5 minutes. Khorasani et al. in Uremia City, Iran reported the response time for traumatic accidents as $5 \mathrm{~min}$ utes [56]. Also, the mean(SD) response time for children in Tehran was reported as 14.9(6.7) minutes by Panahi et al. [57]. Since, arrival to scene is an important factor in quality assessment of pre-hospital care service, this index will be assessed according to a standardized protocol (less than 8 minutes in $80 \%$ of services) [58].

In the current study, $68.4 \%$ of response times were less than 8 minutes. Previous studies reported $81.1 \%$ in Yazd city and $72.5 \%$ in Uremia City $[56,39]$. Panahi et al. found the value of $8.5 \%$ standard response time for the age group below 15 years that had internal problems in Tehran [59]. Thus, it seems that with regard to the lack of heavy traffic in Arak City, Arak EMS could plan to improve this time to reach the standard time of the country.

In this study, the means for time intervals were 16.9 (medi$\mathrm{an}=16 \mathrm{~min}$ ) for the time at the scene, 9.1 (median= $6 \mathrm{~min}$ ) for transfer time and 35.3 (median=31.7min) minutes for total time. Kleindorfer found that "time at the scene" and "transfer time" for stroke and transient ischemic attack patients were 14.1 and 13.1 minutes, respectively [43]. Another study that conducted by Bidari et al. to evaluate the performance of pre-hospital care services in patients transported to Hazrat-e Rasoul-e Akram Hospital, reported "transfer time" and "total time" as 34 and 47 minutes, respectively [32]. According to some authors, reported "total time" was 71 minutes (Puolakka et al.). Other reported values were 6.1, 6.3, and 29.2 minutes for the "time at the scene", "transfer time", and "total time", respectively for motor accidents in Uremia city $[56,60]$.

Results from Table 3 showed that the "time at the scene" value was higher in patients with correct diagnosis by EMS technicians than that value for patients with wrong or without diagnosis. However, no statistically significant differences were found between these groups. Also the mean transfer time in patients with correct diagnosis was very different (3.9 vs. 9.1 to $12.1 \mathrm{~min}$ ). The mean total time from dispatch to arrival at a hospital was 35.7 minutes for patients with correct diagnosis and there were no statistically significant differences with other patients (wrong or without diagnosis).

In another study by Ramanujam et al. time intervals (response time, transfer time, and total time) were reported as 6,19 , and 39 minutes for patients with correct diagnosis and 5, 20, and 40 minutes for other patients [61]. Therefore, our results agree with other studies in this subject. Luca and colleagues investigated transfer time after and before training course for technicians on stroke and reported its decline from 31.8 to 35.8 minutes [62]. Harbison et al. reported that an accurate diagnosis could assist in rapid transfer to appropriate medical center. Results from these studies are similar to our results [34].

\section{Conclusion}

The results of this study showed that the correct diagnosis by EMS technicians could result in faster transfer of the patient to appropriate hospital. Our data revealed that accuracy of stroke diagnosis in EMS technicians was low, also it has inverse relationship with the time of transfer to hospital. Currently, there is not any standard tool for EMS technicians to diagnose stroke.

Many studies showed that the use of diagnostic tools in pre-hospital phase can improve accuracy of diagnosis and reduce transfer time to hospital. It is recommended that more appropriate pre-hospital diagnosis tools of stroke be developed and handed to EMS staff, for more accurate diagnosis of strokes and their better treatment.

\section{Acknowledgments}

We acknowledge assistance of management and staff of Valiasr Hospital and Arak EMS. This research was supported and granted by University of Social Welfare 
\& Rehabilitation Sciences with code No. 348 in 2012. Finally, authors wish to thank all dedicated people who participated in this study.

\section{Conflict of Interests}

The authors declared no conflict of interests.

\section{References}

[1] Mousavi LS, Lotfi R, Dadkhah Tehrani T, Abedini Z, Khorami A. [Risk factor frequencies in ischemic and hemorrhagic stroke: a comparative study (Persian)]. Journal of Shahid Beheshti School of Nursing \& Midwifery. 2009; 19(64):12-17.

[2] Edlow JA, Selim MH. Neurology Emergencies. New York: Oxford University Press; 2011.

[3] Goldstein LB, Bushnell CD, Adams RJ, Appel LJ, Braun LT, Chaturvedi $S$, et al. Guidelines for the primary prevention of stroke a guideline for healthcare professionals from the American Heart Association. Stroke. 2011; 42(2):517-84.

[4] Deakin CD, Alasaad M, King P, Thompson F. Is ambulance telephone triage using advanced medical priority dispatch protocols able to identify patients with acute stroke correctly? Emergency Medicine Journal. 2009; 26(6):442-45.

[5] Bowman S, Jethro De Lisle. Emergency Cardiac and Stroke Care in Washington. Olympia, Washington D.C.: Washington State Department of Health; 2008.

[6] Qiu Y, Li S. Stroke: coping strategies and depression among Chinese caregivers of survivors during hospitalization. Journal of Clinical Nursing. 2008; 17(12):1563-573.

[7] Brainin M, Heiss WD, Heiss S. Textbook of stroke medicine. New York: Cambridge University Press; 2010.

[8] Dalvandi A, Ekman SL, Maddah SS, Khankeh HR, Heikkilä K. Post stroke life in Iranian people: used and recommended strategies. Iranian Rehabilitation Journal. 2009; 7(1):17-24

[9] Hosseini AA, Sobhani-Rad D, Ghandehari K, Benamer HT. Frequency and clinical patterns of stroke in Iran-systematic and critical review. BMC Neurology. 2010; 10(1):72. doi 10.1186/1471-2377-10-72

[10] Summers D, Leonard A, Wentworth D, Saver JL, Simpson J, Spilker JA, et al. Comprehensive overview of nursing and interdisciplinary care of the acute ischemic stroke patient a scientific statement from the American Heart Association. Stroke. 2009; 40(8):2911-944.

[11] Salman-Roghani R, Delbari A, Tabatabae SS. [Stroke rehabilitation: Principles, advances, early experiences, and realities in Iran (Persian)]. Journal of Sabzevar University of Medical Sciences. 2012; 19(2):96-108.

[12] National Center for Chronic Disease Prevention and Health Promotion, Division for Heart Disease and Stroke Prevention. Stroke fact sheet [Internet]. 2016 [cited 2016 June 16]; Available from: http://www.cdc.gov/dhdsp/data_statistics/fact_sheets/fs_stroke.htm
[13] Bray JE, Martin J, Cooper G, Barger B, Bernard S, Bladin C. An interventional study to improve paramedic diagnosis of stroke. Prehospital Emergency Care. 2005; 9(3):297-302.

[14] Buettner JR. Fast facts for the ER nurse: neurological emergency. New York: Springer; 2010.

[15] Mitchel E, Medzon R. Emergency medicine. Lippincott: Williams \& wilkins; 2005.

[16] American Heart Association. Part 9: adult Stroke, hospital (Box 2). Dallas, T.X.: American Heart Association; 2005. doi: 10.1161/circulationaha.105.166562

[17] Gladstone DJ, Rodan LH, Sahlas DJ, Lee L, Murray BJ, Ween JE, et al. A citywide prehospital protocol increases access to stroke thrombolysis in Toronto. Stroke. 2009; 40(12):3841-844.

[18] Mistovich J, Karren K, Hafen B. Prehospital emergency care [R. Shahrami, S. Khoramnia, A. Shahrami, F. Rezvani, H Soltani, M. Abozari, et al., Persian trans]. $4^{\text {th }}$ ed. Tehran: Simindokht Pub; 2010 .

[19] Kurz MW, Kurz KD, Farbu E. Acute ischemic stroke; from symptom recognition to thrombolysis. Acta Neurologica Scandinavica. 2013; 127(196):57-64.

[20] National Stroke Association. Stroke 101: fast facts on stroke: National Stroke Association [Internet]. 2014 [cited 2016 Feb. 12]. Available from: http://www.stroke.org/sites/default/ files/resources/NSA_\%20FactSheet_Stroke_101_2014.pdf

[21] Iqbal A. The "golden hour" treatment of acute ischemic stroke. Medicine \& Health. 2011; 94(12):378-95.

[22] McGruder HF, Croft JB, Zheng ZJ. Characteristics of an illdefined diagnosis for stroke opportunities for improvement. Stroke. 2006; 37(3):781-89.

[23] Adams HP, Del Zoppo G, Alberts MJ, Bhatt DL, Brass L, Furlan A, et al. Guidelines for the early management of adults with ischemic stroke. Circulation. 2007; 115(20):478-534.

[24] American Heart Association. Part 7: The era of reperfusion. Circulation. Dallas, T.X.: American Heart Association; 2000. doi: 10.1161/01.CIR.102.suppl_1.I-172

[25] Kue R, Steck A. Prehospital diagnosis and management of patients with a cute stroke. Emergency Medicine Clinics of North America. 2012; 30(3):617-35

[26] Haghani F, Sadeghi N. [Training in pre-hospital emergency: needs and truths (Persian)]. Iranian Journal of Medical Education. 2011; 10(5):1273-279.

[27] Abdullah AR, Smith EE, Biddinger PD, Kalenderian D, Schwamm LH. Advance hospital notification by EMS in acute stroke is associated with shorter door-to-computed tomography time and increased likelihood of administration of tissueplasminogen activator. Prehospital Emergency Care. 2008; 12(4):426-31

[28] Ingolfsson A, Budge S, Erkut E. Optimal ambulance location with random delays and travel times. Health Care Management Science. 2008; 11(3):262-74.

[29] Llanes JN, Kidwell CS, Starkman S, Leary MC, Eckstein M Saver JL. The Los Angeles Motor Scale (LAMS): a new measure to characterize stroke severity in the field. Prehospital Emergency Care. 2004; 8(1):46-50. 
[30] Bray JE, Coughlan K, Barger B, Bladin C. Paramedic diagnosis of stroke examining long-term use of the Melbourne Ambulance Stroke Screen (MASS) in the field. Stroke. 2010; 41(7):1363-366.

[31] Wojner AW, Morgenstern L, Alexandrov AV, Rodriguez D, Persse D, Grotta JC. Paramedic and emergency department care of stroke: baseline data from a citywide performance improvement study. American Journal of Critical Care. 2003; 12(5):411-17.

[32] Bidari A, Abbasi S, Farsi D, Saeidi H, Mofidi M, Radmehr $\mathrm{M}$, et al. [Quality assessment of prehospital care service in patients transported to Hazrat-e- Rasoul Akram hospital (Persian)]. Medical Journal of Tabriz University of Medical Sciences. 2007; 29(3):43-46.

[33] Chenkin J, Gladstone DJ, Verbeek PR, Lindsay P, Fang J Black SE, et al. Predictive value of the Ontario prehospital stroke screening tool for the identification of patients with acute stroke. Prehospital Emergency Care. 2009; 13(2):153-59.

[34] Harbison J, Hossain O, Jenkinson D, Davis J, Louw SJ, Ford GA. Diagnostic accuracy of stroke referrals from primary care, emergency room physicians, and ambulance staff using the face arm speech test. Stroke. 2003; 34(1):71-76.

[35] Ramanujam P, Guluma KZ, Castillo EM, Chacon M, Jensen $\mathrm{MB}$, Patel E, et al. Accuracy of stroke recognition by emergency medical dispatchers and paramedics - San Diego experience. Prehospital Emergency Care. 2008; 12(3):307-13.

[36] Jauch EC, Cucchiara B, Adeoye O, Meurer W, Brice J, Gentile N, et al. Part 11: adult stroke 2010 American Heart Association Guidelines for cardiopulmonary resuscitation and emergency cardiovascular care. Circulation. 2010; 122(3):81828.

[37] Acker JE, Pancioli AM, Crocco TJ, Eckstein MK, Jauch EC, Larrabee $\mathrm{H}$, et al. Implementation strategies for emergency medical services within stroke systems of care: a policy statement from the American Heart Association/American Stroke Association Expert Panel on Emergency Medical Services Systems and the Stroke Council. Stroke. 2007; 38(11):3097-115.

[38] Zweifler RM, York D, Mendizabal JE, Rothrock JF. Accuracy of paramedic diagnosis of stroke. Journal of Stroke and Cerebrovascular Diseases. 1998; 7(6):446-48.

[39] Bahrami M, Ezzatabadi M, Maleki A, Askari R, Ahmadi Tehrani G. [A survey on the Yazd prehospital emergency medical services performance assessment, 2009-2010 (Persian)]. Toloo-E-Behdasht. 2011; 9(4):45-59.

[40] Statistical Center of Iran. [Iran SCo (Persian)]. 2011. Available from: http://www.amar.org.ir/Portals/2/pdf/jamiat_ shahrestan_keshvar3.pdf

[41] [Arak city (Persian)] [Internet]. 2004 [Cited 2004 March 25]. Available from: http://www.tebyan.net/newindex. aspx?pid $=20854$

[42] Altintas KH, Bilir N. Ambulance times of Ankara emergency aid and rescue services' ambulance system. European Journal of Emergency Medicine. 2001; 8(1):43-50.

[43] Kleindorfer DO, Lindsell CJ, Broderick JP, Flaherty ML Woo D, Ewing I, et al. Community socioeconomic status and prehospital times in acute stroke and transient ischemic attack do poorer patients have longer delays from 911 call to the emergency department? Stroke. 2006; 37(6):1508-513.
[44] Lacy CR, Suh DC, Bueno M, Kostis JB. Stroke collaborative study group. Delay in presentation and evaluation for acute stroke time registry for outcomes knowledge and epidemiology. Stroke. 2001; 32(1):63-69.

[45] Mosley I, Nicol M, Donnan G, Patrick I, Dewey H. Stroke symptoms and the decision to call for an ambulance. Stroke. 2007; 38(2):361-66.

[46] Zhang S, Hu D, Wang X, Yang J. Use of emergency medical services in patients with acute myocardial infarction in China. Clinical Cardiology. 2009; 32(3):137-41. doi: 10.1002/clc.20247

[47] Nagaraja N, Bhattacharya P, Mada F, Salowich-Palm L, Hinton S, Millis S, et al. Gender based differences in acute stroke care in Michigan hospitals. Journal of the Neurological Sciences. 2012; 314(1):88-91.

[48] Kothari R, Barsan W, Brott T, Broderick J, Ashbrock S. Frequency and accuracy of prehospital diagnosis of acute stroke. Stroke. 1995; 26(6):937-41.

[49] Fonarow GC, Reeves MJ, Zhao X, Olson DM, Smith EE, Saver JL, et al. Age-related differences in characteristics, performance measures, treatment trends, and outcomes in patients with ischemic stroke. Circulation. 2010; 121(7):879-91.

[50] Frendl DM, Strauss DG, Underhill BK, Goldstein LB. Lack of impact of paramedic training and use of the cincinnati prehospital stroke scale on stroke patient identification and onscene time. Stroke. 2009; 40(3):754-56.

[51] Halpern M, Meier N, Fischer U, Haefeli T, Kappeler L, Brekenfeld C, et al. Age-dependent differences in demographics, risk factors, co-morbidity, etiology, management, and clinical outcome of acute ischemic stroke. Journal of Neurology. 2008; 255(10):1503-507.

[52] Chen RL, Balami JS, Esiri MM, Chen LK, Buchan AM. Ischemic stroke in the elderly: an overview of evidence. Nature Reviews Neurology. 2010; 6(5):256-65.

[53] Andjelic SL. Accuracy of prehospital diagnosis of stroke. Czechoslovakia Neuroscience \& Neurosurgery. 2012; 75(1):62-8.

[54] Smith WS, Isaacs M, Corry MD. Accuracy of paramedic identification of stroke and transient ischemic attack in the field. Prehospital Emergency Care. 1998; 2(3):170-5.

[55] Sayre MR. Damage control: past, present, and future of prehospital stroke management. Emergency Medicine Clinics of North America. 2002; 20(4):877-86.

[56] Khorasani-Zavareh D, Bigdeli M, Mohammadi R. Prehospital care time intervals among victims of road traffic injuries in Iran: a cross-sectional study. BMC Public Health. 2010; 10(1):406. doi: 10.1186/1471-2458-10-406

[57] Panahi Farzad K, Azizabadi Faraahani M, Khodami Vishteh H, Asaari S. [Time indices of pediatric prehospital emergency care in Tehran, 2006 (Persian)]. Journal of Iran University Of Medical Sciences. 2008; 15(58): 69-80.

[58] Iran Laws. [Collection of laws in Ministry of Health and Medical Education (Persian)]. Tehran: Payam-e Edalat; 2009.

[59] Panahi F, Mohebbi HA, Farahani MA, Vishteh HRK, Assari S. [Prehospital emergency service for internal medicine problems in pediatrics; causes, time indices and outcomes. Iranian Journal of Pediatrics. 2007; 17(2):45-53. 
[60] Puolakka T, Väyrynen T, Häppölä O, Soinne L, Kuisma $\mathrm{M}$, Lindsberg PJ. Sequential analysis of pretreatment delays in stroke thrombolysis. Academic Emergency Medicine. 2010;17(9):965-69.

[61] Ramanujam P, Castillo E, Patel E, Vilke G, Wilson MP, Dunford JV. Prehospital transport time intervals for acute stroke patients. Journal of Emergency Medicine. 2009; 37(1):40-45.

[62] Luca AD, Toni D, Lauria L, Sacchetti ML, Rossi PG, Ferri $\mathrm{M}$, et al. An emergency clinical pathway for stroke patientsresults of a cluster randomised trial. BMC Health Services Research. 2009; 9(1):2. doi: 10.1186/1472-6963-9-14 
